\title{
Functional influences on orienting a reference frame
}

\author{
LAURA A. CARLSON-RADVANSKY and ZHIHUA TANG \\ University of Notre Dame, Notre Dame, Indiana
}

\begin{abstract}
Spatial relational terms like above are defined with respect to a reference frame. Reference frames are imposed on reference objects and define the space in which to search for a located object. Reference frames have a number of parameters that must be set during spatial term assignment, including origin, scale, and orientation. Previous research has shown that a functional relationship between objects in the scene influences the selection of a reference frame (Carlson-Radvansky \& Radvansky, 1996). However, because this work operated at the level of the reference frame, generalizing across various spatial terms, it is unclear how function had its influence. One possibility is that function influences how the parameters of a reference frame are set. Consistent with this idea, Carlson-Radvansky, Covey, and Lattanzi (1999) showed an influence of function on where the origin of a reference frame was imposed. The present paper demonstrates that function also influences how the orientation of the axes of a reference frame is set. The implications for setting multiple parameters of a reference frame are discussed.
\end{abstract}

Everyday tasks often require spatially locating one object with respect to another, such as placing a coffee mug under a coffee pot. In English, we can describe the spatial relationship between these objects with locative prepositions, such as below, as in The mug is below the coffee pot. Spatial terms specify the location of one object (the located object) by referring to the known location of a second object (the reference object). In the above sentence, coffee pot is a reference object, and mug is a located object. According to many theories of spatial relations, finding the located object involves starting at the reference object, moving in the direction specified by the spatial relation (e.g., below), and searching the surrounding space for the located object (Logan \& Sadler, 1996; see also CarlsonRadvansky \& Irwin, 1994; Carlson-Radvansky \& Logan, 1997; Garnham, 1989; Herskovits, 1986; Levelt, 1984).

The direction indicated by a spatial relation is defined with respect to a reference frame. For projective spatial relations, such as above and below, a reference frame is defined as a set of three coordinate orthogonal axes whose intersection point is the origin (Miller \& Johnson-Laird, 1976). Reference frames have a number of parameters, including scale, orientation, and direction (Logan \& Sadler, 1996). Scale defines units of distance. Orientation specifies whether a given axis corresponds to the above/ below, front/back, or left/right dimension. Direction defines the endpoint of each axis (e.g., which endpoint is above on the vertical above/below axis).

These parameters are not fixed but can be set according to various sources of information. This results in three different types of reference frames (Farah, Brunn, Wong,

This work was supported in part by NSF Grant SBR97-27638. We thank Melissa Byerly, Natalie Hupf, Erin Lum, and Dasia Webster for help with data collection and Eric Covey for help throughout the project. Correspondence concerning this article should be addressed to L. A. Carlson-Radvansky, Department of Psychology, University of Notre Dame, Notre Dame, IN 46556 (e-mail: laura.c.radvansky.2@nd.edu).
Wallace, \& Carpenter, 1990; Fillmore, 1975; Friederici \& Levelt, 1990; Hinton \& Parsons, 1988; Levinson, 1996; Miller \& Johnson-Laird, 1976). Using Levinson's taxonomy, the relative reference frame is based on the viewer of the scene, the intrinsic reference frame is based on the reference object, and the absolute reference frame is based on salient properties of the environment.

Given different reference frames for defining spatial relations, much research has focused on identifying factors that influence the selection of a reference frame. Some of these factors include the presence of a functional relationship between the located and the reference objects (Carlson-Radvansky \& Radvansky, 1996), coordination between speaker and listener (Schober, 1993), the size of the environment being described (Taylor \& Tversky, 1996), and aspects of the individual objects in the scene, such as movement (Fillmore, 1975; Levelt, 1982). The goal of the present paper is to determine more precisely how a functional relationship between the objects influences the apprehension of spatial relations.

\section{FUNCTIONAL INFLUENCES ON REFERENCE FRAME SELECTION}

Carlson-Radvansky and Radvansky (1996) tested the idea that a functional relationship between the located and the reference objects would influence the type of reference frame that was selected to describe the spatial relationship between the objects. The function of an object has been shown to significantly influence processing in naming and categorization tasks (e.g., Landau, Smith, \& Jones, 1998; Miller \& Johnson-Laird, 1976; Rosch, Mervis, Gray, \& Boyes-Braem, 1976), speeded classification and part detection (Lin \& Murphy, 1997), and the probability of encoding in language and memory (Radvansky \& Copeland, in press). Moreover, Herskovits (1986) has suggested that the function of an object mediates the acceptable use of a spatial relation to describe spatial 
configurations involving that object. For example, according to Herskovits, one can say "the potato in the dish," but not "the potato in the tray." Although a tray and a dish have fundamentally the same shape, the different functions that these objects fulfill (containment vs. support) lead to different conceptualizations of the objects and, hence, to a difference in the applicability of the spatial relation in to describe the spatial configuration.

Carlson-Radvansky and Radvansky (1996) tested whether object function would influence the manner in which spatial configurations were described by building on the suggestion that spatial terms defined by the intrinsic reference frame focus attention on the objects and are thus more functional (Miller \& Johnson-Laird, 1976; Ullmer-Ehrich, 1982). If so, the presence of a functional relationship between the located and the reference objects should result in a preference for describing the objects' spatial layout with intrinsic terms. To test this prediction, Carlson-Radvansky and Radvansky showed participants pictures of functionally related objects in a typically interactive spatial configuration (e.g., a mail carrier facing a mailbox). The intrinsic reference frame described the spatial configuration between the objects with a particular spatial term (i.e., front), but the relative/absolute reference frame described the same configuration with a different spatial term (i.e., left). The participants rated sentences containing intrinsic terms as more acceptable descriptions of the pictures than were sentences containing relative/absolute relations, even though both descriptions could be used. In contrast, when the participants were shown pictures containing the same objects in a noninteractive configuration (e.g., a mail carrier facing away from a mailbox), they rated sentences containing the relative/ absolute relations as more acceptable than sentences containing intrinsic relations. Note that because the same objects were used, the change in preference for selecting a reference frame was due to a change in the depicted interaction between the objects. Similarly, when the participants were shown pictures containing unrelated objects in a potentially interactive configuration (e.g., a mail carrier facing a birdhouse), they rated sentences containing relative/ absolute relations as more acceptable than sentences containing intrinsic relations. Note that because the same configuration was used, this change in preference can be attributable to a change in the identity of the objects. Thus, there seem to be two sources of functional influence on reference frame selection: whether reference and located objects are presented in a typically interactive manner and whether they are related.

\section{FUNCTIONAL INFLUENCES ON SETTING THE PARAMETER OF A REFERENCE FRAME}

Carlson-Radvansky and Radvansky (1996) examined the influence of function on reference frame selection at the level of the reference frame, showing general preferences for using one reference frame over another without regard to the particular terms being used. Given the design of the experiments, an analysis of how function had its influence was not possible. For example, the assignment of spatial relations to reference frames was not balanced. The set of intrinsic terms used to describe the pictures included above, front, and behind; however, the set of relative/absolute terms used to describe the pictures included above, left, right, and below. Moreover, the pairings of the terms were not crossed. For example, intrinsic above was always paired with relative/absolute left. As such, any analysis across reference frames necessarily occurs across the different axes of the reference frames and across different endpoints of these axes. This makes it difficult to isolate the locus of the effect from underlying preferences for using different axes and different endpoints.

The goal of the present paper was to test the idea that function has its influence in setting the parameters of a reference frame. Recently, Carlson-Radvansky, Covey, and Lattanzi (1999) showed that function influenced the placement of the origin of the reference frame. In their Experiment 2, they presented subjects with pictures of a piggy bank (the reference object). Across trials, a coin (the located object) was placed in various locations around the piggy bank. Subjects rated the acceptability of the relation above as a description of the relationship between the coin and the piggy bank. This methodology elicits a spatial template (Logan \& Sadler, 1996), a parsing of space into good, acceptable, and bad regions corresponding to the applicability of the spatial relation. The good region is generally considered the best use of the spatial relation and corresponds to placements along the axis of the reference frame. As such, the peak of the template can be used as an index to the location of the reference frame. The critical manipulation was the location of a functional part of the piggy bank (its slot). Across subjects, it could appear at the back, in the middle, or at the front of the bank. The results were that the peak in the spatial template corresponded to the location of the slot. For subjects with the slot in the back of the piggy bank, the highest ratings corresponded to placements of the coin over the back of the piggy bank. For subjects with the slot in the middle of the piggy bank, the highest ratings corresponded to placements of the coin over the middle of the piggy bank. For subjects with the slot in the front of the bank, the highest ratings corresponded to placements of the coin over the front of the piggy bank. Assuming that the peak represents placements along the axis of a reference frame, these data indicate that the function of the reference object influences where the origin of the reference frame is imposed (for further discussion, see Carlson, in press).

Object effects can also influence how the scale of the reference frame is used. Morrow and Clark (1988) showed that interpretation of the verb approach was sensitive to the nature of the interaction between the located and the reference objects. The critical manipulation was the size of the objects. They presented subjects with such sentences as "The mouse/tractor is approaching the gate/fence." The 
task was to estimate the distance between the two objects. Distance estimates were directly influenced by the size of the objects and their presumed zone of interaction. Preliminary work in my laboratory has extended this finding to the relations front, back, left, and right. Thus, these projective relations convey a distance that is influenced by the size of the objects and their potential interaction (Covey \& Carlson, 2000).

The goal of the present paper is to examine whether function influences the setting of the orientation parameter of a reference frame. To test this, in the present experiment, the orientation of the vertical axes of the intrinsic and the relative/absolute reference frames were dissociated by rotating the reference object. Preferences for using the spatial relation above with respect to these orientations were collected. If function biases the orientation of a reference frame, placements with respect to the intrinsic reference frame should be preferred to placements with respect to the relative/absolute reference frame. This follows the logic used by Carlson-Radvansky and Radvansky (1996), in which a manipulation that emphasizes the interaction between the objects should elicit a preference for the intrinsic reference frame. In contrast to the Carlson-Radvansky and Radvansky study that made comparisons across different axes (horizontal vs. vertical) and different endpoints of a reference frame, the design of the present experiment permits comparison across orientations of the same axis (vertical) and the same endpoint (e.g., above), thereby successfully isolating the orientation parameter of the reference frame.

Moreover, above is an interesting relation to use in an investigation of the orientation parameter. Garnham (1989) has claimed that only absolute definitions of above are acceptable, whereas Levelt (1984) suggested that intrinsic definitions are also possible. Carlson-Radvansky and Irwin (1993) used a number of tasks to show that people prefer to use the absolute reference frame to define above, with a smaller but nonetheless significant preference for using the intrinsic reference frame (see also Friederici \& Levelt, 1990). Although some manipulations increased the acceptability of the intrinsic reference frame (e.g., decreasing the distance between the objects, or adding other objects in the scene that were also rotated), in all cases, the absolute reference frame remained more strongly preferred. These results stand in contrast to the present prediction of a stronger preference for the intrinsic reference frame than for the relative/absolute reference frame in the presence of a functional relationship between the objects. Consistent with this prediction, Coventry and Prat-Sala (1998) have recently argued that above may be sensitive to functional influences, although perhaps not as strongly as other relations, such as over.

\section{THE PRESENT EXPERIMENT}

To test the idea that function influences the setting of the orientation parameter, the participants were shown sentence-picture pairs and were asked to rate the accept- ability of the sentence as a description of the picture. As is shown in Figure 1, three types of pictures were used. Panel A shows a picture containing functionally related reference and located objects (a hamburger and a mustard bottle) placed in a typical interactive configuration; this is the related/interactive condition. Panel B shows a picture containing functionally related reference and located objects in a noninteractive configuration; this is the related/noninteractive condition. Panel $\mathrm{C}$ shows a picture containing functionally unrelated reference and located objects (a hamburger and a bottle of ant killer) placed in the same relative orientations as those in panel A; this is the unrelated condition. Note that it is possible for the pair of objects in the unrelated condition to functionally interact. For example, someone with malicious intent may spray ant killer on the hamburger in order to poison the hamburger's consumer. For most of the pairs of objects, a plausible interaction could be envisioned. However, because an interaction for a small set of pairs seemed unlikely, we refer to this condition as unrelated, rather than as unrelated/interactive. All the participants saw pictures in the related/interactive condition. In addition, half of the participants saw pictures in the related/noninteractive condition, and half saw pictures in the unrelated condition. Comparison of the related/interactive condition to the related/noninteractive condition allows an assessment of whether the possible interaction between the objects contributes to the functional influence. Comparison of the related/interactive condition and the unrelated condition allows an assessment of whether object relatedness contributes to the functional influence.

To evaluate preferences for using the intrinsic or the relative/absolute reference frame, five types of spatial configurations of reference and located objects were used; these are illustrated in Figure 2, using a functionally related/ interactive picture. For the two configurations in panel A, the reference object was upright in a canonical orientation; thus, the intrinsic reference frame was perfectly aligned with the absolute and relative reference frames, yielding only one definition of above. For these configurations, the located object could be placed either above (Placement 1 ) or not above (Placement 2 ) the reference object. Ratings of sentences containing the term above for these pictures should be very high and very low, respectively; as such, these are control conditions. For the other three configurations in panel $\mathrm{B}$, the reference and located objects were rotated $90^{\circ}$ into a noncanonical orientation, thereby dissociating the intrinsic reference frame from the coincident relative and absolute reference frames. Thus, for these configurations there were two possible definitions of above: intrinsic and relative/absolute. Accordingly, the located object could be placed above the reference frame according to the relative/absolute reference frames (Placement 3 ), above according to the intrinsic reference frame (Placement 4 ), or not above the reference object according to any reference frame (Placement 5). The predictions were that ratings for intrinsic placements of the located object would be greater for the functionally related/interactive pictures 
A

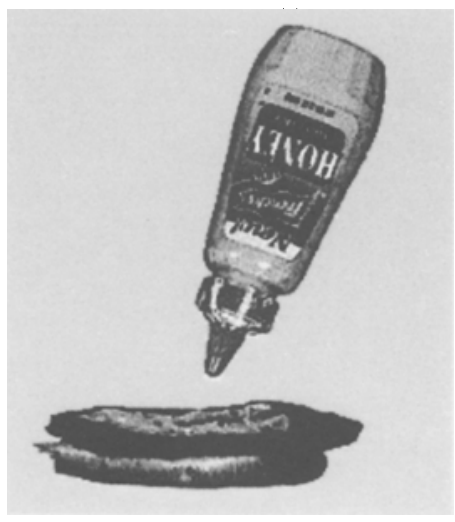

B

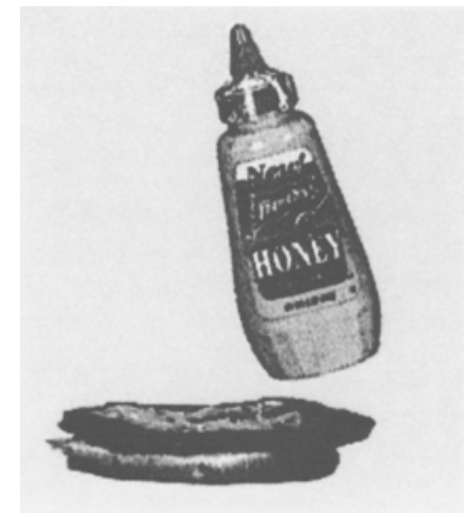

C

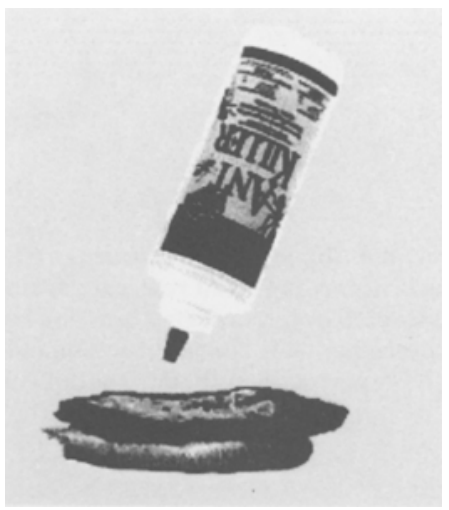

Figure 1. Examples of the three picture types containing a hamburger as the reference object and a mustard bottle (panels $A$ and $B$ ) or a bottle of ant killer (panel $C$ ) as the located object. Panel A shows the related/interactive condition, panel B shows the related/noninteractive condition, and panel $C$ shows the unrelated condition. See the text for details. than for either the related/noninteractive or the unrelated pictures. In addition, intrinsic placements should be preferred over relative/absolute placements for the functionally related/interactive pictures.

\section{METHOD}

\section{Participants}

Eighty University of Notre Dame undergraduates participated in exchange for course credit or for a monetary reimbursement of $\$ 6 / \mathrm{h}$. Forty students were randomly assigned to the related/interactive and the related/noninteractive pictures, and 40 were assigned to the related/interactive and the unrelated pictures. All the participants gave informed consent and were naïve with respect to the hypotheses.

\section{Stimuli}

Color photographs of 40 objects were clipped from various magazines, catalogs, and fliers. For each reference object, a pair of objects from the same sources was also selected to serve as the located objects. Within a pair, the objects were matched in size and shape. One object in the pair was functionally related to the reference object, so that the typical interaction between the objects involved placing one object above the other object (e.g., a hamburger and a mustard bottle, a candle and a candlestick, and a mop and a bucket). The other object was unrelated to the reference object (e.g., a bottle of ant killer and a hamburger, a carrot and a candlestick, and a rake and a bucket). A complete list of reference and located objects appears in the Appendix.

All the photographs were scanned into a Dell Pentium computer and placed on a gray field. For each reference object, 15 pictures were created that contained the reference object and one of the located objects in some spatial configuration; these corresponded to the 3 relatedness conditions $\times 5$ configurations. Ten lists were created that counterbalanced the configurations across the related/ interactive pictures and the related/noninteractive pictures. As shown in Figure 1, panels A and B, the same located objects were used in these pictures, but they were placed in different orientations. Ten other lists were created that counterbalanced the configurations across the related/interactive pictures and the unrelated pictures. As shown in Figure 1, panels A and C, different located objects were used, but they were placed in the same orientation. Thus, each participant saw only one type of nonfunctional picture: related/ noninteractive or unrelated. Within each stimulus list, each reference object and located object were displayed only once, and each participant saw four trials (using different objects) in each of the 10 conditions. Four participants were randomly assigned to each of the lists.

The pictures were displayed as 64 color bitmaps during the experiment. Accompanying the picture was a sentence of the form "The located object is above the reference object," with the appropriate names of the located object and the reference object filled in. Below the sentence was a reminder of the rating scale, with 1 marked as not at all acceptable and 7 marked as perfectly acceptable.

\section{Procedure}

The participants were told that they would be seeing sentence-picture pairs and that the task was to rate the acceptability of the sentence as a description of the picture on a scale ranging from 1 to 7. Each participant performed 40 trials in a random order, and the experiment lasted about $15 \mathrm{~min}$.

\section{RESULTS}

Because each participant saw functional pictures (related/interactive) with only one type of nonfunctional picture (the related/noninteractive pictures or the unre- 
A

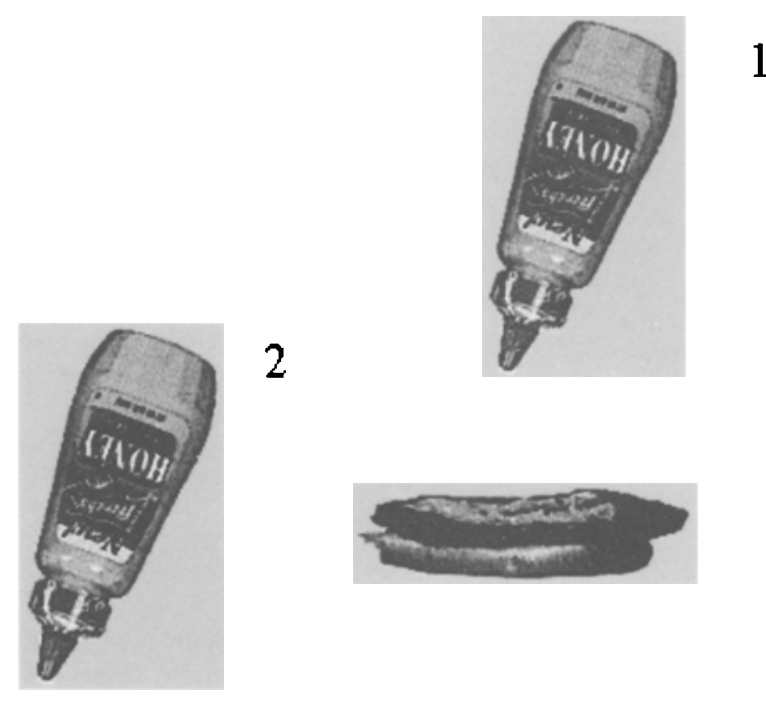

B
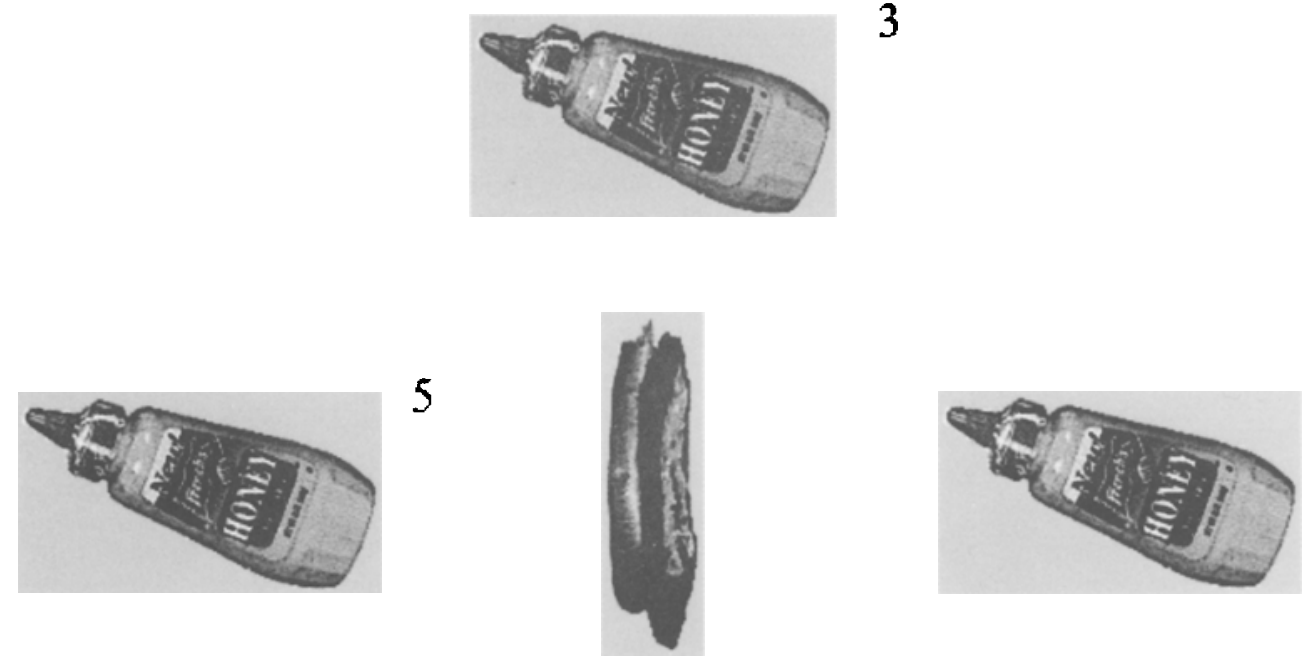

Figure 2. The five possible spatial configurations of the mustard bottle (located object) and hamburger (reference object). Panel $A$ shows the reference object in canonical orientation. Placement 1 is above according to all reference frames. Placement 2 is not above according to all reference frames. Panel $B$ shows the reference and located objects rotated into noncanonical orientation. Placement 3 is above according to the relative/absolute reference frames. Placement 4 is above according to the intrinsic reference frame. Placement 5 is not above according to all reference frames. In the experiment, only one spatial configuration was presented on each trial.

lated pictures), the data will be presented broken down by type of nonfunctional picture. Analyses with participants as the random factor are subscripted with 1; analyses with items as the random factor are subscripted with 2. A significance level of $p<.05$ was adopted unless otherwise noted. Significant interactions were further explored by calculating $95 \%$ confidence intervals based on the error term of the interaction (Fisher, 1966; Loftus \& Masson, 1994).

\section{Related/Interactive and \\ Related/Noninteractive Pictures}

Mean acceptability ratings as a function of spatial configuration and relatedness are shown in Table 1. 
Table 1

Mean Acceptability Ratings for

Related/Interactive and Related/Noninteractive Pictures by Placements of the Located Object

\begin{tabular}{lcc}
\hline & \multicolumn{2}{c}{ Functional Relatedness } \\
\cline { 2 - 3 }$\quad$ Placements & $\begin{array}{c}\text { Related } \\
\text { Interactive }\end{array}$ & $\begin{array}{c}\text { Related } \\
\text { Noninteractive }\end{array}$ \\
\hline Canonical orientation & 6.8 & \\
$\quad$ Above & 2.0 & 6.1 \\
$\quad$ Not above & & 2.2 \\
Noncanonical orientation & 4.9 & \\
Intrinsic above & 4.1 & 4.2 \\
Relative/absolute above & 1.9 & 4.2 \\
Not above & 2.0 \\
\hline
\end{tabular}

Reference object in canonical orientation. Acceptability ratings were submitted to a 2 (relatedness) $\times 2$ (placement: above or not above) repeated measures analysis of variance (ANOVA). There was a main effect of relatedness $\left[F_{1}(1,39)=4.7, M S_{\mathrm{e}}=0.6 ; F_{2}(1,39)=5.0\right.$, $\left.M S_{\mathrm{e}}=0.8\right]$, a main effect of placement $\left[F_{1}(1,39)=579.8\right.$, $\left.M S_{\mathrm{e}}=1.3 ; F_{2}(1,39)=706.7, M S_{\mathrm{e}}=1.2\right]$, and a significant interaction $\left[F 1(1,39)=22.6, M S_{\mathrm{e}}=0.4 ; F_{2}(1,39)=\right.$ $\left.8.9, M S_{\mathrm{e}}=1.1\right]$. Generally, above placements were rated as highly acceptable, and not-above placements were rated as not acceptable. On the basis of a critical difference of .3 for significance, the form of the interaction was significantly higher ratings for the above placement of the located object for the related/interactive pictures $(M=6.8)$ than for the related/noninteractive pictures $(M=6.1)$. Note that this change in preference is surprising, given that the located object is placed above with respect to all reference frames across both conditions. This difference thus reflects a preference for depicting objects in their typical interaction. There was no difference in acceptability for the not-above placements for related/interactive pictures $(M=$ $2.0)$ and related/noninteractive pictures $(M=2.2)$.

Reference object in noncanonical orientation. Acceptability ratings were submitted to a 2 (relatedness) $\times 3$ (placement: relative/absolute above, intrinsic above or not above) repeated measures ANOVA. There was a main effect of relatedness by participants $\left[F_{1}(1,39)=7.5\right.$, $\left.M S_{\mathrm{e}}=0.3\right]$, but not by items $\left[F_{2}(1,39)=1.6, M S_{\mathrm{e}}=1.0\right.$, $p=.21]$, a main effect of placement $\left[F_{1}(2,78)=30.1\right.$, $\left.M S_{\mathrm{e}}=1.3 ; F_{2}(2,78)=152.1, M S_{\mathrm{e}}=1.0\right]$, and an interaction that was significant by participants $\left[F_{1}(2,78)=8.1\right.$, $\left.M S_{\mathrm{e}}=0.5\right]$ and marginally significant by items $\left[F_{2}(2,78)=\right.$ 2.6, $\left.M S_{\mathrm{e}}=1.6, p=.08\right]$. For follow-up tests of the interaction, a critical difference of 3 was required for significance.

Looking across functional and nonfunctional pictures, intrinsic above placements of the located object were rated significantly higher for the related/interactive pictures $(M=4.9)$ than for the related/noninteractive pictures $(M=4.2)$. However, there was no difference in acceptability for the relative/absolute placements $(M \mathrm{~s}=4.1$ and 4.2 for the related/interactive and related/noninteractive pictures, respectively) or for the not-above placements $(M \mathrm{~s}=1.9$ and 2.0 for the related/interactive and related $/$ noninteractive pictures, respectively). Looking across types of reference frames, intrinsic placements $(M=4.9)$ were preferred over relative/absolute placements $(M=$ 4.1) for the related/interactive pictures; however, there was no difference in preference between the intrinsic placements $(M=4.2)$ and the relative/absolute placements $(M=4.2)$ for the related/noninteractive pictures. Thus, a functional interaction between related objects increased preference for the intrinsic reference frame.

\section{Related/Interactive and Unrelated Pictures}

Mean acceptability ratings as a function of spatial configuration and relatedness are shown in Table 2.

Reference object in canonical orientation. Acceptability ratings were submitted to a 2 (relatedness) $\times 2$ (placement: above or not above) repeated measures ANOVA. There was a main effect only of placement $\left[F_{1}(1,39)=724.2, M S_{\mathrm{e}}=1.2 ; F_{2}(1,39)=1045.7, M S_{\mathrm{e}}=\right.$ 0.8 ; for all other effects, $F<1.1, p>.31$ ]. Generally, above placements were rated as highly acceptable, and not-above placements were rated as not acceptable.

Reference object in noncanonical orientation. Acceptability ratings were submitted to a 2 (relatedness) $\times$ 3 (placement: relative/absolute above, intrinsic above or not above) repeated measures ANOVA. There was a main effect of placement $\left[F_{1}(2,78)=65.4, M S_{\mathrm{e}}=3.3\right.$; $\left.F_{2}(2,78)=167.8, M S_{\mathrm{e}}=1.3\right]$ and an interaction between relatedness and placement that was significant by participants $\left[F_{\mathrm{l}}(2,78)=4.2, M S_{\mathrm{e}}=0.4\right]$, but not by items $\left[F_{2}(2,78)=2.1, M S_{\mathrm{e}}=0.8, p=.13\right]$. For follow-up tests of the interaction, a critical difference of 3 was required for significance.

Looking across functional and nonfunctional pictures, intrinsic above placements of the located object were rated significantly higher for the related/interactive pictures $(M=4.8)$ than for the unrelated pictures $(M=4.5)$. There was no difference in acceptability for the relative/absolute placements $(M \mathrm{~s}=4.2$ and 4.3 for the related/interactive and unrelated pictures, respectively), but the not-above placements were more acceptable for the unrelated pictures $(M=1.8)$ than for the related/interactive pictures $(M=1.5)$. Looking across type of reference frame, intrinsic placements $(M=4.8)$ were preferred over relative/ absolute placements $(M=4.2)$ for the functionally related/

\section{Table 2}

Mean Acceptability Ratings for

Related/Interactive and Unrelated Pictures by Placements of the Located Object

\begin{tabular}{lcc}
\hline \multicolumn{1}{c}{ Placements } & \multicolumn{2}{c}{ Functional Relatedness } \\
\cline { 2 - 3 } Canonical orientation & Related/Interactive & Unrelated \\
$\quad$ Above & 6.5 & \\
$\quad$ Not above & 1.7 & 6.5 \\
Noncanonical orientation & & 1.9 \\
$\quad$ Intrinsic above & 4.8 & \\
Relative/absolute above & 4.2 & 4.5 \\
Not above & 1.5 & 4.3 \\
\hline
\end{tabular}


interactive pictures; however, there was no significant difference in preference for intrinsic placements $(M=4.5)$ and relative/absolute placements $(M=4.3)$ for the unrelated pictures. Thus, functional relatedness increased preference for the intrinsic reference frame.

\section{DISCUSSION}

The results demonstrate an increased preference for using the intrinsic reference frame to define above when the reference object and the located object were functionally related and depicted in an interactive configuration. More specifically, intrinsic placements were considered more acceptable for related/interactive pictures than for either related/noninteractive or unrelated pictures. These findings are consistent with the idea that the intrinsic reference frame emphasizes the objects and their relationship (Miller \& Johnson-Laird, 1976; UllmerEhrich, 1982). This pattern extends the results of CarlsonRadvansky and Radvansky (1996) by showing that the functional influence operates on the orientation parameter of the reference frame, biasing it to be set with respect to the reference object, with a consequent reliance on the intrinsic reference frame.

These results also support the idea that there are two sources of the influence of function: the relatedness of the objects and the depicted interaction. Of these two, the depicted interaction seems stronger. For example, the change in preference for the intrinsic reference frame over the relative/absolute reference frames was stronger when the interaction between the objects was manipulated than when object relatedness was manipulated (cf. Tables 1 and 2). This could be because most of the unrelated objects could potentially interact with the reference object, thereby boosting preference for the intrinsic reference frame in the unrelated condition. As such, the function of the reference object, independent of the identity of the located object, seems of primary importance. Additional support for this idea was recently obtained by Carlson-Radvansky et al. (1999). Specifically, in their Experiment 1, Carlson-Radvansky et al. presented pictures of located and reference objects to participants and asked them to place the located object above the reference object. They found that participants were biased to place both related and unrelated located objects toward the functional part of the reference object, with this bias stronger for related than for unrelated objects.

Another important finding of the present experiment was that intrinsic placements were preferred over relative/ absolute placements for the related/interactive pictures. This stands in contrast to the manipulations used by Carlson-Radvansky and Irwin (1993) that increased preference for use of the intrinsic frame but never exceeded preference for the relative/absolute reference frames. This underscores the inherent flexibility in setting these parameters and the need to understand the factors that influence how these parameters are set. It is interesting to note that the preference for the use of the relative/absolute ref- erence frames remained relatively constant across all types of pictures (see also Carlson-Radvansky \& Radvansky, 1996, where changes in preferences for the intrinsic reference frame were responsible for the pattern of data). This makes sense in that the manipulations used in the present experiment were designed to emphasize the objects and their relations; as such, they should, in principle, only affect the intrinsic reference frame, and not the relative or the absolute reference frame. These findings support the idea that particular factors may interact with particular reference frames.

Note, too, that the use of particular term may also interact with particular reference frames. For example, Franklin, Henkel, and Zengas (1995) showed that the mapping of front onto space around one's own body (i.e., the relative reference frame) is quite different than the mapping of front onto space surrounding a model's body (i.e., the intrinsic reference frame). Thus, front is interpreted differently across the different reference frames. This points toward the need to systematically identify the factors that influence how the various parameters of a reference frame are set.

Finally, by necessity, this work isolated the functional relationship between the objects to determine whether it would significantly influence how the orientation of a reference frame was set. The results indicate sensitivity to this factor. Nevertheless, the ratings for both intrinsic above and relative/absolute above were intermediate and certainly not as acceptable as ratings for intrinsic/relative/ absolute above with the canonical pictures. Thus, while function can play a role in orienting the axes, the strength of its influence may be mediated by other factors as well. For example, it is possible that contextual information would also bias selection of the intrinsic reference frame, in effect boosting the intermediate rating much higher. Alternatively, coordination issues between speaker and listener (e.g., Schober, 1993) may encourage use of an alternative reference frame, thereby effectively decreasing the intermediate rating. Thus, although many individual factors have been identified as influencing the selection of a reference frame (such as function's orienting the axes of a reference frame, as has been shown here), it certainly is the case that these factors combine in such a way that selection of the particular reference frame in contextrich, face-to-face communication involving spatial relations is relatively unambiguous. The challenge is to figure out how these independent factors combine.

\section{SETTING THE PARAMETERS OF A REFERENCE FRAME}

The results of this experiment illustrate an effect of function on the orientation parameter of a reference frame. Other work has also shown a functional influence on parameter setting, such as where the origin is imposed (Carlson-Radvansky et al., 1999) and how the scale is set (Covey \& Carlson, 2000). Such broad-based influence raises some important questions about how the individ- 
ual parameters of a reference frame are set and, more generally, about how reference frames are classified. That is, if one parameter is set on the basis of a particular source of information, is that same source of information used to set the other parameters? For example, assume that function sets the orientation of the axes in accordance with the reference object. Is the scale of the reference frame also set with respect to the reference object? This would indicate that the parameters are dependent. Alternatively, the parameters may be independent, so that each is set in accordance with a preferred source of information. For example, orientation may be set with respect the reference object if the function of the object is particularly salient, but scale may be set with respect to units based on the distance between the viewer and the reference object. If so, one parameter would be set with respect to the intrinsic reference frame, whereas another parameter would be set with respect to the relative reference frame. Finally, note that such independence would have consequences for classification. If parameters can be set independently, what is the basis for classifying reference frames with respect to one parameter (e.g., orientation), when other parameters may be defined with respect to different sources of information?

In summary, the results of this experiment suggest (1) that function influences how the orientation of a reference frame is set, $(2)$ that preferences for using particular spatial relations are extremely flexible and that different factors may affect different reference frames, and (3) that understanding how the parameters of a reference frame are set is critical to understanding reference frame selection.

\section{REFERENCES}

CARLSON, L. A. (in press). Object use and object location: The effect of function on spatial relations. In E. van der Zee \& U. Nikanne (Eds.), Cognitive interfaces. Oxford: Oxford University Press.

Carlson-Radvansky, L. A., Covey, E., \& Lattanzi, X. (1999). "What" effects on "where": Functional influences on spatial relations. Psychological Science, 10, 516-521.

CARLSON-RAdvansky, L. A., \& IRWIN, D. E. (1993). Frames of reference in vision and language: Where is above? Cognition, 46, 223-244.

Carlson-Radvansky, L. A., \& IRwin, D. E. (1994). Reference frame activation during spatial term assignment. Journal of Memory \& Language, 33, 646-671

Carlson-Radvansky, L. A., \& Logan, G. D. (1997). The influence of reference frame selection on spatial template construction. Journal of Memory \& Language, 37, 411-437.

Carlson-Radvansky, L. A., \& Radvansky, G. A. (1996). The influence of functional relations on spatial term selection. Psychological Science, 7, 56-60.

Coventry, K. R., \& Prat-Sala, M. (1998). Geometry, function, and the comprehension of over, under, above and below. In M. A. Gernsbacher \& S. J. Derry (Eds.), Proceedings of the Twentieth Annual
Conference of the Cognitive Science Society (pp. 261-266). Mahwah, $\mathrm{NJ}$ : Erlbaum.

Covey, E. S., \& Carlson, L. A. (2000). How far is near? Examining the distance conveyed by spatial terms. Manuscript submitted for publication.

Farah, M. J., Brunn, J. L., Wong, A. B., Wallace, M. A., \& CarPENTER, P. A. (1990). Frames of reference for allocating attention to space: Evidence from the neglect syndrome. Neuropsychologia, $\mathbf{2 8}$, 335-347.

Fillmore, C. J. (1975). Santa Cruz lectures on deixis. Bloomington: Indiana University Linguistics Club.

FisHer, R. A. (1966). The design of experiments (8th ed.). Edinburgh: Oliver \& Boyd.

Franklin, N., Henkel, L. A., \& Zengas, T. (1995). Parsing surrounding space into regions. Memory \& Cognition, 23, 397-407.

Friederici, A. D., \& LEvelt, W. J. M. (1990). Spatial reference in weightlessness: Perceptual factors and mental representations. Perception \& Psychophysics, 47, 253-266.

Garnham, A. (1989). A unified theory of meaning of some spatial relational terms. Cognition, 31, 45-60.

HERSKOVITS, A. (1986). Language and spatial cognition: An interdisciplinary study of the prepositions of English. Cambridge: Cambridge University Press.

Hinton, G. E., \& Parsons, L. M. (1988). Scene-based and viewercentered representations for comparing shapes. Cognition, 30, 1-35.

LANDAU, B., Smith, L., \& Jones, S. (1998). Object shape, object function, and object name. Journal of Memory \& Language, 38, 1-27.

LeVelt, W. J. M. (1982). Cognitive styles in the use of spatial direction terms. In R. J. Jarvella \& W. Klein (Eds.), Speech, place and action (pp. 251-268). Chichester, U.K.: Wiley.

LEVELT, W. J. M. (1984). Some perceptual limitations on talking about space. In A. J. van Doorn, W. A. van der Grind, \& J. J. Koenderink (Eds.), Limits in perception (pp. 323-358). Utrecht: VNU Science Press.

Levinson, S. (1996). Frames of reference and Molyneux's questions: Cross-linguistic evidence. In P. Bloom, M. A. Peterson, L. Nadel, \& M. Garrett (Eds.), Language and space (pp. 109-169). Cambridge, MA: MIT Press.

LiN, E. L., \& MURPHY, G. L. (1997). Effects of background knowledge on object categorization and part detection. Journal of Experimental Psychology: Human Perception \& Performance, 23, 1153-1169.

LoFTUS, G. R., \& MAsson, M. E. J. (1994). Using confidence intervals in within-subject designs. Psychonomic Bulletin \& Review, 1, 476-490.

LogAN, G. D., \& SADLER, D. D. (1996). A computational analysis of the apprehension of spatial relations. In P. Bloom, M. A. Peterson, L. Nadel, \& M. Garrett (Eds.), Language and space (pp. 493-529). Cambridge, MA: MIT Press.

Miller, G. A., \& Johnson-LaIRD, P. N. (1976). Language and perception. Cambridge, MA: Harvard University Press.

MoRROW, D. G., \& ClARK, H. H. (1988). Interpreting words in spatial descriptions. Language \& Cognitive Processes, 3, 275-291.

Radvansky, G. A. \& Copeland, D. E. (in press). Functionality and spatial relations in memory and language. Memory \& Cognition.

Rosch, E., Mervis, C., Gray, W., Johnson, D., \& Boyes-Braem, P. (1976). Basic objects in natural categories. Cognitive Psychology, $\mathbf{8}$, 382-439.

SCHOBER, M. (1993). Spatial perspective-taking in conversation. Cognition, 47, 1-24.

TAYloR, H. A., \& TVERSKY, B. (1996). Perspective in spatial descriptions. Journal of Memory \& Language, 35, 371-391.

ULLMER-EHRICH, V. (1982). The structure of living space descriptions. In R. J. Jarvella \& W. Klein (Eds.), Speech, place and action (pp. 219249). Chichester, U.K.: Wiley. 
APPENDIX

\begin{tabular}{|c|c|c|}
\hline \multicolumn{3}{|c|}{ APPENDIX } \\
\hline Reference Object & $\begin{array}{c}\text { Related } \\
\text { Located Object }\end{array}$ & $\begin{array}{c}\text { Unrelated } \\
\text { Located Object }\end{array}$ \\
\hline Television & antenna & lawn ornament \\
\hline VCR & remote & phone \\
\hline Man's head & hat & dustpan \\
\hline Bowl with eggs & whisk & brush \\
\hline Nail & hammer & pipe \\
\hline Glass & gallon of milk & liquid detergent \\
\hline Screw in table & screwdriver & baster \\
\hline Ashtray & cigarette & chalk \\
\hline Printer & printout & magazine \\
\hline Toaster & bread & sponge \\
\hline Mousepad & mouse & seashell \\
\hline Podium & microphone & binoculars \\
\hline Clay pot & plant & feather duster \\
\hline Hard drive & monitor & framed picture \\
\hline Piggy bank & coin & gear-wheel \\
\hline Ink bottle & pen & laser pointer \\
\hline Toothbrush & toothpaste & caulking tube \\
\hline Wine glass & wine bottle & bowling pin \\
\hline Plastic cup & straw & pencil \\
\hline Recycle bin & soda can & slinky \\
\hline Kitchen fire & extinguisher & water bottle \\
\hline Can & can opener & pruner \\
\hline Pedestal & statue & bed post \\
\hline Yogurt & spoon & popsicle stick \\
\hline Shirt & clothes pin & tweezer \\
\hline Tea cup & kettle & watering can \\
\hline Bucket & mop & bamboo rake \\
\hline Tripod & camera & Walkman \\
\hline Vase & flowers & pom pom \\
\hline Tape deck & cassette & floppy disk \\
\hline Datebook & pen & candy cane \\
\hline Pizza board & pizza & record \\
\hline Cotton candy maker & cotton candy & balloon \\
\hline Lamp & lightbulb & avocado \\
\hline Workbench & drill & pistol \\
\hline Candlestick & candle & carrot \\
\hline Hamburger & mustard & pesticide \\
\hline Telephone & receiver & bar bell \\
\hline Lighter & cigar & beef jerky stick \\
\hline Ironing board & iron & hand mixer \\
\hline
\end{tabular}

(Manuscript received November 4, 1998;

revision accepted for publication September 10, 1999.) 